\title{
Antibiotic Resistance Patterns of Pseudomonas spp. Isolated from the River Danube
}

\author{
Clemens Kittinger ${ }^{1}$, Michaela Lipp ${ }^{1}$, Rita Baumert ${ }^{1}$, Bettina Folli ${ }^{1}$, Günther Koraimann ${ }^{2}$, \\ Daniela Toplitsch ${ }^{1,2}$, Astrid Liebmann ${ }^{1,2}$, Andrea J. Grisold ${ }^{1}$, Andreas H. Farnleitner ${ }^{3,4}$, \\ Alexander Kirschner ${ }^{3,5}$ and Gernot Zarfel ${ }^{1 *}$ \\ ${ }^{1}$ Institute of Hygiene, Microbiology and Environmental Medicine, Medical University Graz, Graz, Austria, ${ }^{2}$ Institute of \\ Molecular Biosciences, University of Graz, Graz, Austria, ${ }^{3}$ Interuniversity Cooperation Centre for Water and Health, Vienna, \\ Austria, ${ }^{4}$ Research Group Environmental Microbiology and Molecular Ecology, Institute of Chemical Engineering, Vienna \\ University of Technology, Vienna, Austria, ${ }^{5}$ Institute for Hygiene and Applied Immunology, Medical University of Vienna, \\ Vienna, Austria
}

Spread and persistence of antibiotic resistance pose a severe threat to human health, yet there is still lack of knowledge about reservoirs of antibiotic resistant bacteria in the environment. We took the opportunity of the Joint Danube Survey 3 (JDS3), the world's biggest river research expedition of its kind in 2013, to analyse samples originating from different sampling points along the whole length of the river. Due to its high clinical relevance, we concentrated on the characterization of Pseudomonas spp. and evaluated the resistance profiles of Pseudomonas spp. which were isolated from eight sampling points. In total, 520 Pseudomonas isolates were found, 344 (66.0\%) isolates were identified as Pseudomonas putida, and 141 (27.1\%) as Pseudomonas fluorescens, all other Pseudomonas species were represented by less than five isolates, among those two $P$. aeruginosa isolates. Thirty seven percent (37\%) of all isolated Pseudomonas species showed resistance to at least one out of 10 tested antibiotics. The most common resistance was against meropenem (30.4\%/158 isolates) piperacillin/tazobactam (10.6\%/55 isolates) and ceftazidime (4.2\%/22 isolates). 16 isolates (3.1\%/16 isolates) were multi-resistant. For each tested antibiotic at least one resistant isolate could be detected. Sampling points from the upper stretch of the River Danube showed more resistant isolates than downriver. Our results suggest that antibiotic resistance can be acquired by and persists even in Pseudomonas species that are normally not in direct contact with humans. A possible scenario is that these bacteria provide a reservoir of antibiotic resistance genes that can spread to related human pathogens by horizontal gene transfer.

Keywords: JDS3, Pseudomonas, antibiotic resistance, water, Danube

\section{INTRODUCTION}

Multiresistant bacteria are present in many surface waters (Girlich et al., 2011; Czekalski et al., 2012; Tissera and Lee, 2013; Blaak et al., 2015; Maravic et al., 2015). Typically, evidence is provided through fecal indicators, and mostly relates to short river sections or sampling at individual points. Thus, investigations of whole water systems are rare, especially if the river 
passes through 10 riparian countries, like the River Danube. Water samples from the third Joint Danube Survey (JDS3), the world's biggest river research expedition of its kind, offered a chance for evaluating resistance of bacteria over a whole river system. Based on these samples, a resistance profile of Pseudomonas spp. over the course of the multinational River Danube was created in our study.

Pseudomonas species can be naturally found in all surface waters, lakes and rivers, but they are rarely found in drinking water. Pseudomonas spp. can survive in both low and high nutrition environments (Mena and Gerba, 2009) or even in double distilled water and, in addition, can help Salmonellae survive in this environment (Warburton et al., 1994). The whole group of non-fermenting Gram negative bacilli is suspected of establishing the basis for multiresistance in Gram negative bacteria, as the members of this group carry multiple intrinsic resistances and have the ability to acquire and evolve additional resistances (Farinas and Martinez-Martinez, 2013). Pseudomonas species are known to harbor multiple intrinsic and acquired resistance genes, host several mobile genetic elements, and also exchange them with other families of Gram negative bacilli like Enterobacteriaceae (Juan Nicolau and Oliver, 2010; Pfeifer et al., 2010). Hence Pseudomonas are known starting points of several important carbapenemases families (Pfeifer et al., 2010). The occurrence and spread of carbapenemases have become a substantial global health problem, as they inactivate a substantial antibiotic class.

The most common pathogen in this genus is Pseudomonas aeruginosa. It causes a variety of different infections, from easyto-cure ear infections, serious infections of burn patients, to severe lung infections which lead to major complications in cystic fibrosis patients (Barbier and Wolff, 2010; Azzopardi et al., 2014). Besides Pseudomonas aeruginosa, other species e.g., Pseudomonas putida or Pseudomonas fluorescens are also a cause for infections in clinical settings (Gilarranz et al., 2013; Erol et al., 2014; Bhattacharya et al., 2015; Mazurier et al., 2015).

The aim of the study was to evaluate the resistance profiles of Pseudomonas spp. isolated at selected sites along the whole course of the River Danube. Pseudomonas spp. were chosen for various reasons: they belong to the native bacterial community in surface waters, they are clinically relevant, and changes in their natural resistance profiles indicate anthropogenic influence. This study, therefore, aims at monitoring the presence of resistances of Pseudomonas spp. to clinically important antibiotics along the river course. Doing so, changes in the resistance profiles were to be detected, if possible.

\section{MATERIALS AND METHODS \\ Sample Collection}

Samples were collected during the JDS 3, which was organized by the International Commission for the Protection of the Danube River (ICPDR).The ICPDR is a transnational body, which has been established to implement the Danube River Protection Convention. All Danube countries are member states of the ICPDR on the base of the "Convention on Cooperation for the
Protection and Sustainable use of the Danube River" (Danube River Protection Convention).

Between Aug. 12 and Sep. 26, 2013, surface water samples for microbiological investigations were collected from 68 sampling sites along the river Danube (JDS 3, 2015).

For each sampling site, water samples were taken at three sampling points, on the left, in the middle and on the right side of the River Danube. Samples were collected in sterile 1-L glass flasks, from $30 \mathrm{~cm}$ below the river surface (Figure 1, pink and violet dots, high resolution map is added as Supplementary Figure S1). Duplicate volumes of the samples $(45 \mathrm{ml})$ were filled into sterile non-toxic $50 \mathrm{ml}$ plastic vials (Techno Plastic Products AG, TPP, Switzerland), containing $5 \mathrm{ml}$ glycerol (final conc. $10 \% \mathrm{v} / \mathrm{v}$ ). The vials were shaken and turned around to homogenize glycerol and water and after that immediately stored at $-20^{\circ} \mathrm{C}$ on board of the JDS3 research ship. After transfer to the home laboratory (beginning of October 2013) the samples were stored at $-80^{\circ} \mathrm{C}$. Out of the 68 sampling sites the four sites directly downstream from the cities Vienna, Budapest, Novi Sad, and Bucharest were chosen for investigation. In addition four non-city related sampling sites were chosen (including the delta and near the starting point of the JDS3; Table 1).

\section{Isolation of Bacteria}

The frozen samples were thawed and $15 \mathrm{ml}$ (left, middle, right $5 \mathrm{ml}$ each) were plated in $0.5 \mathrm{ml}$ portions on different selective agars: Endo Agar, Xylose Lysine Deoxycholat Agar (XLD agar), and Chromocult Coliform Agar (CCA), (all Merck, Austria). Growth conditions were $37 \pm 1^{\circ} \mathrm{C}$ for $18-24 \mathrm{~h}$. Identification of Pseudomonas by MALDI-TOF-MS (matrix-assisted laser desorption ionization time-of-flight mass spectrometry) was per-formed as described previously (Jamal et al., 2014). A single bacterial colony was deposited on the target slide, followed by the addition of the matrix (VITEK MS-CHCA) and air drying. Samples were processed in the MALDI-TOFMS spectrometer VITEK ${ }^{\circledR}$ MS (Biomerieux, Austria). Microbial identification was achieved by obtaining the spectra using MALDI-TOF technology and analyzing the spectra with the VITEK MS database. The peaks from these spectra were compared with the characteristic pattern for the species, genus or family of the microorganism, leading to identification of the organism.

\section{Antibiotic Susceptibility Testing}

For all identified Pseudomonas spp., antibiotic susceptibility testing was performed as recommended by the European Committee on Antimicrobial Susceptibility Testing (EUCAST) including recommended controls. Inhibition zone diameters were interpreted according to EUCAST guidelines (http://www.eucast.org/clinical_breakpoints/) (EUCAST, 2013; Matuschek et al., 2014). Classification of multiresistance of Pseudomonas spp. was evaluated according to the Robert Koch Institut (RKI, Germany, http://www.rki. de/EN/Home/homepage_node.html). The suspension for inoculation was prepared from an over-night pure culture on a blood agar (non-selective medium). Colonies were picked with 


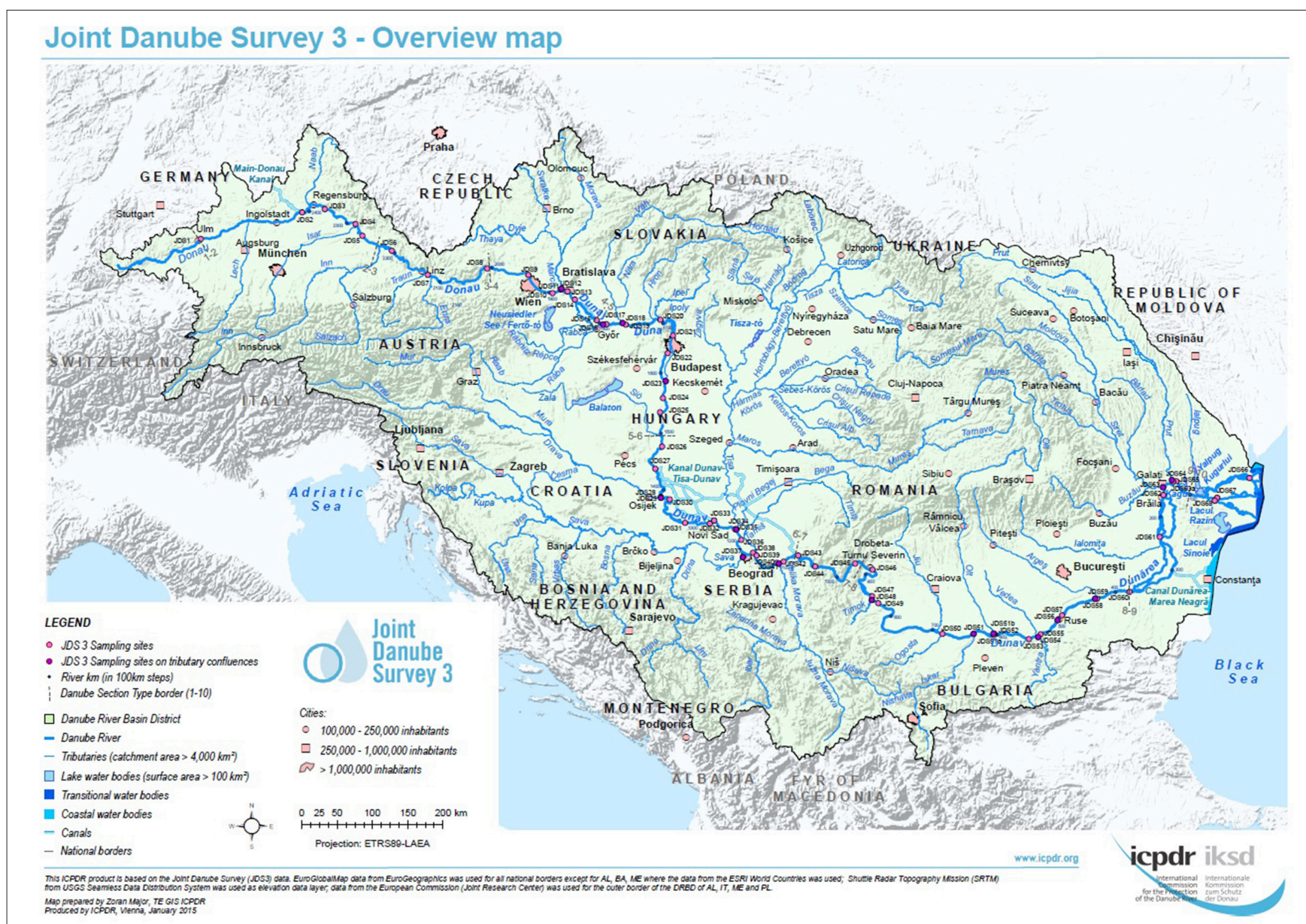

FIGURE 1 | Overview of the JDS3 sampling points along the river Danube. The map was taken with kind permission of the ICPDR.

(http://www. danubesurvey.org/results).

TABLE 1 | Investigated sampling sites names and numbers according to JDS3, country and detailed location (ds = downstream, us = upstream).

\section{E. coli $[\mathrm{MPN} / 100 \mathrm{ml}]$}

\begin{tabular}{|c|c|c|c|c|c|c|c|}
\hline Site & Name & Country & Location & rkm & Left & Middle & Right \\
\hline JDSO3 & Geisling & Germany & ds Regensburg & 2354 & 1728 & 1739 & 1304 \\
\hline JDS10 & Wildungsmauer & Austria & ds Vienna & 1895 & 1044 & 917 & 1739 \\
\hline JDS28 & US Drava & Croatia/Serbia & us tributary Drava & 1384 & 2445 & 1998 & 2880 \\
\hline JDS36 & DS Tisa, Serbia & Serbia & ds Novi Sad & 1200 & 11060 & 9900 & 4960 \\
\hline JDS68 & St. Gheorge arm & Romania & river delta & 104 & 4880 & 2424 & 2431 \\
\hline
\end{tabular}

Basic microbiological parameters. MPN, most probable numbers; rkm, river kilometer. E. coli concentrations were determined according to ISO 9308-2 (JDS 3, 2015).

*Arges is the river flowing through Bucharest.

a sterile loop and suspended in sterile saline $(0.85 \% \mathrm{NaCl} w / \mathrm{v}$ in water) to the density of a McFarland 0.5 standard (DensiCheck, Biomerieux, Austria). The suspension was then plated on Mueller-Hinton Agar by using an automatic plate rotator (Retro
C80, Biomerieux, Austria). Antibiotic test disks were applied firmly on the agar surface within $15 \mathrm{~min}$ of inoculation of the plates. Plates were incubated at $36^{\circ} \mathrm{C}$ for $16-20 \mathrm{~h}$. After incubation, inhibition zones were measured. 
TABLE 2 | EUCAST Clinical Breakpoints for Pseudomonas spp.

\begin{tabular}{lccc}
\hline Antibiotic & $\begin{array}{c}\text { Antibiotic } \\
\text { centration on } \\
\text { test disk }(\boldsymbol{\mu} \mathbf{g})\end{array}$ & $\begin{array}{c}\text { Susceptibly } \\
\text { inhibition } \\
\text { zone }(\mathbf{m m})\end{array}$ & $\begin{array}{c}\text { Resistant } \\
\text { (including intermediate) } \\
\text { inhibition zone }(\mathbf{m m})\end{array}$ \\
\hline Piperacilin/tazobactam & $30 / 6$ & $\geq 18$ & $<18$ \\
Ceftazidime & 10 & $\geq 16$ & $<16$ \\
Cefepime & 30 & $\geq 19$ & $<19$ \\
Meropenem & 10 & $\geq 18$ & $<18$ \\
Imipenem & 10 & $\geq 20$ & $<20$ \\
Amikacin & 30 & $\geq 24$ & $<24$ \\
Gentamicin & 10 & $\geq 15$ & $<15$ \\
Tobramycin & 10 & $\geq 16$ & $<16$ \\
Ciprofloxacin & 5 & $\geq 25$ & $<25$ \\
Levofloxacin & 5 & $\geq 20$ & $<20$ \\
\hline
\end{tabular}

The following antibiotics were tested:

Piperacilin/tazobactam (TZP), ceftazidime (CAZ), cefepime (FEP), meropenem (MEM), imipenem (IPM), amikacin (AN), gentamicin (GM), tobramycin (NN), ciprofloxacin (CIP), levofloxacin (LEV), and sulfamethoxazole/trimethoprim (SXT) (all Becton Dickinson, Schwechat, Austria; Table 2).

SXT was evaluated because sulfamethoxazole was part of the chemical analysis of the River Danube water. There are no sulfamethoxazole/trimethoprim breakpoints according to EUCAST for Pseudomonas spp. To include the sensitivity of Pseudomonas spp. to sulfamethoxazole/trimethoprim, diameters of inhibition areas were evaluated and compared (SXT test discs specification: sulfamethoxazole/trimethoprim: 1.25/23.75 $\mu \mathrm{g}$ ).

\section{Modified Hodge Test}

To estimate the presence of carbapenemases, a modified Hodge test was performed with all isolates resistant to at least one carbapenem. In brief: after plating of a carbapenem sensitive Klebsiella pneuomoniae (ATCC 700603) a $10 \mu \mathrm{g}$ imipenem or meropenem disc was placed in the center, and each test isolate was streaked from the disk to the edge of the plate. After incubation $\left(37 \pm 1^{\circ} \mathrm{C}\right.$ for $\left.18-24 \mathrm{~h}\right)$ the plates were checked for showing a "cloverleaf shaped" inhibition zone. Isolates that produced carbapenemases enabled growth of the sensitive Klebsiella closer to the antibiotic disk (Bennett et al., 2009).

\section{Data Analyses}

Statistical analyses were calculated with GraphPadPrism ${ }^{\mathrm{TM}} 5.01$ for Windows, GraphPad Software, San Diego California USA, www.graphpad.com.

\section{RESULTS}

\section{Species Composition of Isolates}

In total, 520 Pseudomonas spp. were isolated, the fewest isolates were obtained from JDS68 (32 isolates), and the highest number could be isolated from JDS28 sample with 117 isolates (Table 3). The most abundant Pseudomonas species were Pseudomonas putida (66.0\%/344 isolates) and Pseudomonas fluorescens (27.1\%/141 isolates). Each of the other detected species represented less than $1 \%$ of all isolates (five or fewer isolates). Only two Pseudomonas aeruginosa were isolated, both from JDS28.

\section{Antibiotic Resistances}

Wild type Pseuodomas species are susceptible to all tested antibiotics except SXT (EUCAST, 2013). The highest number of resistances was the one against meropenem with 158 resistant isolates (30.4\%), 55 isolates $(10.6 \%)$ were resistant to piperacilin/tazobactam and 22 isolates (4.2\%) resistant to ceftazidime, $11(2.1 \%)$ to imipenem and four $(0.8 \%)$ to cefepime (Table 3). In the fluoroquinolone group only six (1.2\%) of the isolates showed levofloxacin resistance, whereas 18 (3.4\%) isolates were resistant against ciprofloxacin. Resistance to ciprofloxacin turned out to be the most frequent resistance of all tested non beta-lactam antibiotics in this study. One isolate which was resistant to levofloxacin was still sensitive to ciprofloxacin. Resistance to aminoglycosides was very rare, two isolates were resistant to amikacin and gentamicin respectively and only one isolate showed no susceptibility to tobramycin (Table 3). The 11 isolates resistant to imipenem were also resistant to meropenem and were positive in the modified Hodge test, indicating carbapenemase activity. 327 (62.9\%) isolates were susceptible to all tested antibiotics with EUCAST breakpoints (clinical resistance wild type), 128 (24.6\%) isolates showed resistance to one, and 49 (9.4\%) isolates were resistant to two tested antibiotics. The most common combination of resistances was to meropenem and piperacillin/tazobactam. Sixteen isolates revealed resistance to 3 or more antibiotics, including $12(2.3 \%)$ isolates with three resistances, three $(0.6 \%)$ isolates with four and one $(0.2 \%)$ with five. Eight of them were classified as multiresistant as they were resistant to three or four different antibiotic classes (Table 4).

The 12 isolates with three antibiotic resistances split in eight different resistance patterns, the isolates with four resistances all displayed the same pattern. The two Pseudomonas aeruginosa were susceptible to all tested antibiotics. Out of all other isolated species in this study at least one showed the EUCAST defined antibiotic susceptibility wild type, susceptible to all tested antibiotics (except SXT; Supplementary Table S1).

Four sampling points had less than $50 \%$ isolates with clinical resistance wild type, JDS03, JDS10, JDS22, and JDS59. Three sampling points revealed no isolate that was non-susceptible to three or more tested antibiotics, JDS10, JDS63 and JDS68 (Table 4).

Surprisingly, the upstream sampling points (JDS03, JDS10, JDS22, and JDS28) revealed higher proportions of resistant bacteria than the downstream ones (Figure 2). Sampling point JDS03 revealed the highest proportions of resistance to ceftazidime (28.6\% of JDS03 isolates) and ciprofloxacin (25.7\% of JDS03 isolates). Sampling point JDS22 showed high rates for piperacillin/tazobactam (32.6\% of the JDS22 isolates), meropenem (69.6\% of the JDS22 isolates), and imipenem (13.0\% of the JDS22 isolates).

SXT was chosen for testing as sulfamethoxazole was measured directly during JDS3. Chemical analysis revealed a sampling 
TABLE 3 | Number of isolated Pseudomonas spp. at the investigated sampling points (SP) and the number of resistant isolates to the testes antibioitic.

\begin{tabular}{|c|c|c|c|c|c|c|c|c|c|c|c|}
\hline SP & TZP & CAZ & FEP & MEM & IPM & AN & GM & NN & CIP & LEV & No. Isolates \\
\hline JDS03 & 3 & 10 & 1 & 15 & 0 & 0 & 1 & 0 & 9 & 1 & 35 \\
\hline JDS10 & 2 & 2 & 0 & 16 & 2 & 0 & 0 & 0 & 1 & 0 & 33 \\
\hline JDS22 & 15 & 5 & 0 & 32 & 6 & 1 & 0 & 0 & 2 & 0 & 46 \\
\hline JDS28 & 19 & 4 & 2 & 35 & 3 & 0 & 0 & 0 & 2 & 3 & 117 \\
\hline JDS36 & 6 & 0 & 0 & 10 & 0 & 1 & 1 & 1 & 1 & 1 & 109 \\
\hline JDS59 & 6 & 1 & 1 & 21 & 0 & 0 & 0 & 0 & 0 & 0 & 46 \\
\hline JDS63 & 3 & 0 & 0 & 21 & 0 & 0 & 0 & 0 & 2 & 1 & 102 \\
\hline JDS68 & 1 & 0 & 0 & 8 & 0 & 0 & 0 & 0 & 1 & 0 & 32 \\
\hline Sum & 55 & 22 & 4 & 158 & 11 & 2 & 2 & 1 & 18 & 6 & 520 \\
\hline
\end{tabular}

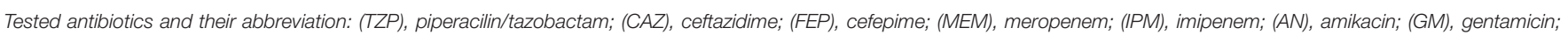
(NN), tobramycin; (CIP), ciprofloxacin; (LEV), levofloxacin.

TABLE 4 | Resistance pattern of isolates showing resistances to three or more of the tested antibiotics.

\begin{tabular}{|c|c|c|c|}
\hline Isolate & Species & Resistance Pattern & Multidrug Resistance (MDR) \\
\hline JDS03PS007 & Pseudomonas fluorescens & CAZ, MEM, CIP & MDR 3 \\
\hline JDS03PS016 & Pseudomonas fluorescens & TZP, CAZ, GM, MEM, CIP & MDR 4 \\
\hline JDS03PS019 & Pseudomonas putida & TZP, MEM, CIP & MDR 3 \\
\hline JDS03PS020 & Pseudomonas fluorescens & CAZ, MEM, CIP & MDR 3 \\
\hline JDS03PS032 & Pseudomonas fluorescens & CAZ, CIP, LEV & \\
\hline JDS22PS016 & Pseudomonas putida & TZP, CAZ, MEM & MDR 3 \\
\hline JDS22PS018 & Pseudomonas fluorescens & CAZ, IMP, MEM & \\
\hline JDS22PS032 & Pseudomonas putida & TZP, IMP, MEM & \\
\hline JDS22PS035 & Pseudomonas putida & TZP, IMP, MEM & \\
\hline JDS22PS043 & Pseudomonas putida & TZP, MEM, CIP & MDR 3 \\
\hline JDS28PS083 & Pseudomonas fluorescens & CAZ, FEP, IMP, MEM & \\
\hline JDS28PS113 & Pseudomonas fluorescens & CAZ, IMP, MEM & \\
\hline JDS28PS115 & Pseudomonas putida & TZP, MEM, CIP, LEV & MDR 3 \\
\hline JDS28PS117 & Pseudomonas putida & TZP, MEM, LEV & MDR 3 \\
\hline JDS36PS036 & Pseudomonas putida & TZP, AN, GM, NN & \\
\hline JDS59PS020 & Pseudomonas fluorescens & TZP, CAZ, FEP & \\
\hline
\end{tabular}

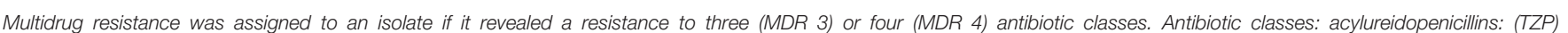

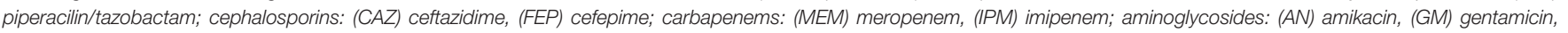
(NN) tobramycin; fluoroquinolones: (CIP) ciprofloxacin, (LEV) levofloxacin.

site (JDS58) with elevated levels for sulfamethoxazole (Arges, tributary) (JDS 3, 2015). The subsequent River Danube sampling site JDS59 did not show significantly reduced diameters $(p=$ 0.68 ) (Figure 3). JDS03 and JDS68 showed both elevated diameters (6.59 mm JDS03, $8.13 \mathrm{~mm}$ JDS68) but only JDS68 differed significantly from the other sampling points $(p<0.05)$.

\section{DISCUSSION}

The spread of antibiotic resistant bacteria, their distribution, and their reservoirs in the environment are important issues. Within the last years many different possible sources have been intensively investigated to shed light on the spread of antibiotic resistant bacteria. Farming and the spread of liquid manure are known to contribute to the spread of multiresistant bacteria (Sengupta et al., 2011; Friese et al., 2013). Recently, the focus has been put on waste water, as bacteria of all kind and with all possible genetic features are mixed up there. And a very critical feature in waste water is the possibility that bacteria harboring resistance exchange their resistance determinants with other bacteria (Korzeniewska and Harnisz, 2013; Reinthaler et al., 2013; Amador et al., 2015). Microorganisms from these and other sources can be relatively easily flushed into surface waters (Czekalski et al., 2012; Zurfluh et al., 2013; Hess and Gallert, 2014), but except for a few studies on relatively small rivers that deal with this topic, the fate of deposited bacteria is quite unclear.

The distribution of susceptible and resistant Pseudomonas spp. at the investigated JDS3 sampling points showed sitespecific differences. At the upstream sampling points, there was a trend to more resistant bacteria (JDS03, JDS10, JDS22, JDS28), and multiresistant Pseudomonas spp. could only be detected in this part of the river. One reason for this finding 


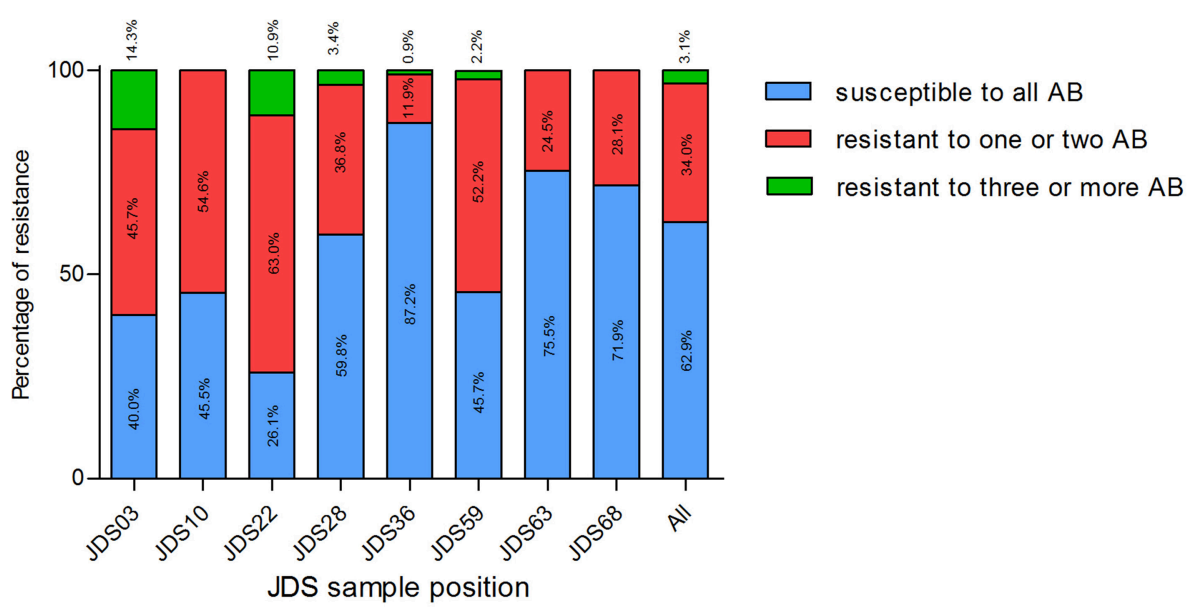

FIGURE 2 | Proportion of isolates susceptible to all tested antibiotics (blue bars), resistant to one or two tested antibiotics (red bars) and resistant to three or more tested antibiotics (green bars), at different sampling points. JDS03, JDS28, JDS63, and JDS68 are non-urban sampling sites, whereas JDS10 is ds Vienna, JDS22 ds Budapest, JDS36 ds Novi Sad and JDS59 is ds Bucharest. Kruskal-Wallis test revealed a non-Gaussian distribution with a $p$ < 0.0001.

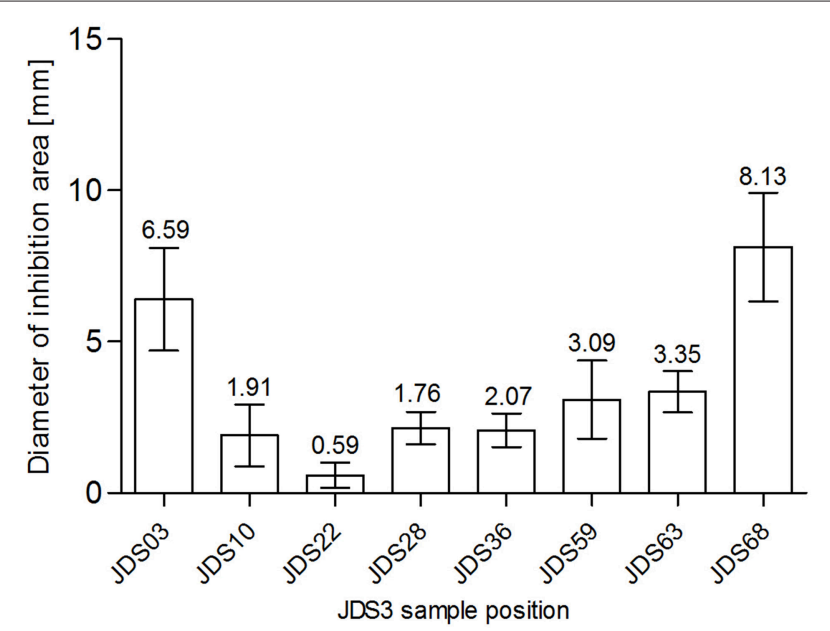

FIGURE 3 | Zone of inhibition diameters $(\mathrm{mm})$ for SXT at the investigated sampling sites. Bars show mean values (values given on top of the bars, $\mathrm{mm}$ ) and standard deviation errors bars (SD error bars) for each sampling site.

could be the lower river water volume in the upstream parts of the River Danube, which might result in less dilution of the resistant microorganisms. Downstream from the cities of Vienna, Budapest, and Bucharest (JDS10, JDS22, and JDS59 via Argeş; Figure 3), the occurrence of resistant bacterial isolates was also elevated, with anthropogenic influence very likely being the reason for that.

Under similar non-selective isolation conditions Suzuki et al. found no resistance to meropenem, gentamicin, amikacin, or ciprofloxacin (Suzuki et al., 2013). A presence of only 8 (1.5\%) multiresistant Pseudomonas spp. present in three of the eight sampling points seems to be a low number. But still, if we extrapolate the number of 8 multiresistant Pseudomonaceae in a collected volume of $75 \mathrm{ml}$ at the 5 sampling points $(5 \times 15 \mathrm{ml})$ to 1 liter, we might estimate over 100 multiresistant bacteria of the Pseudomonas group in one liter of Danube water.

The isolates all over the course of the river Danube showed high resistance rates against meropenem (9.2-69.6\%). The resistance against carbapenems in Pseudomonas spp. is mostly mediated via efflux pumps (intrinsic resistance), especially in water environment (Tacao et al., 2015). However, carbapenem resistance poses a challenge for therapy, regardless of the underlying mechanism. For example, Pseudomonas putida (66\% of all isolates in this study) is increasingly involved in hospital infections (Kim et al., 2012; Molina et al., 2014). These infections come up with severe complications and high mortality rates (up to 40\%). In most of these cases multiresistant Pseudomonas putida was the reason for the infection or the nosocomial outbreak (Kim et al., 2012).

This study is the first study investigating bacterial resistance in a transnational river survey $(2500 \mathrm{rkm})$. Although it was limited by a small sample volume and a fixed time course, the results of this study substantiate the occurrence of waterborne Pseudomonas spp. with non-wild type resistance pattern in the whole River Danube. Their presence and their distribution suggest human influence.

\section{AUTHOR CONTRIBUTIONS}

CK study design, laboratory work, manuscript preparation, data analysis, ML laboratory work, data conservation. RB laboratory work. BF laboratory work. GK data analysis, manuscript preparartion. DT laboratory work, data analysis. AL laboratory work. AG manuscript preparation. AF manuscript preparation. AK sample collection, manuscript preparation. GZ study design, laboratory work, data analysis, manuscript preparation. 


\section{FUNDING}

The Joint Danube Survey was organized by the International Commission for the Protection of the Danube River (ICPDR). The study was supported by the Austrian Science Fund (FWF), project nr P25817-B22.

\section{ACKNOWLEDGMENTS}

We would like to thank Georg Reischer, Stefan Jakwerth, and Stoimir Kolarevic for their help in sampling.

\section{REFERENCES}

Amador, P. P., Fernandes, R. M., Prudencio, M. C., Barreto, M. P., and Duarte, I. M. (2015). Antibiotic resistance in wastewater: occurrence and fate of Enterobacteriaceae producers of class A and class C beta-lactamases. J. Environ. Sci. Health. A. Tox. Hazard. Subst. Environ. Eng. 50, 26-39. doi: 10.1080/10934529.2015.964602

Azzopardi, E. A., Azzopardi, E., Camilleri, L., Villapalos, J., Boyce, D. E., Dziewulski, P., et al. (2014). Gram negative wound infection in hospitalised adult burn patients-systematic review and metanalysis-. PLoS ONE 9:e95042. doi: 10.1371/journal.pone.0095042

Barbier, F., and Wolff, M. (2010). Multi-drug resistant Pseudomonas aeruginosa: towards a therapeutic dead end? Med. Sci. (Paris) 26, 960-968. doi: $10.1051 / \mathrm{medsci} / 20102611960$

Bennett, J. W., Herrera, M. L., Lewis, J. S. II., Wickes, B. W., and Jorgensen, J. H. (2009). KPC-2-producing Enterobacter cloacae and pseudomonas putida coinfection in a liver transplant recipient. Antimicrob. Agents Chemother. 53, 292-294. doi: 10.1128/AAC.00931-08

Bhattacharya, D., Dey, S., Kadam, S., Kalal, S., Jali, S., Koley, H., et al. (2015). Isolation of NDM-1-producing multidrug-resistant Pseudomonas putida from a paediatric case of acute gastroenteritis, India. New Microbes New Infect. 5, 5-9. doi: 10.1016/j.nmni.2015.02.002

Blaak, H., Lynch, G., Italiaander, R., Hamidjaja, R. A., Schets, F. M., and de Roda Husman, A. M. (2015). Multidrug-resistant and extended spectrum betalactamase-producing Escherichia coli in Dutch Surface Water and Wastewater. PLoS ONE 10:e0127752. doi: 10.1371/journal.pone.0127752

Czekalski, N., Berthold, T., Caucci, S., Egli, A., and Burgmann, H. (2012). Increased levels of multiresistant bacteria and resistance genes after wastewater treatment and their dissemination into lake geneva, Switzerland. Front. Microbiol. 3:106. doi: $10.3389 /$ fmicb. 2012.00106

Erol, S., Zenciroglu, A., Dilli, D., Okumus, N., Aydin, M., Gol, N., et al. (2014). Evaluation of nosocomial blood stream infections caused by Pseudomonas species in newborns. Clin. Lab. 60, 615-620. doi: 10.7754/Clin.Lab.2013.130325

EUCAST (2013). European Committee on Antimicrobial Susceptibility Testing (EUCAST). Växjö.

Farinas, M. C., and Martinez-Martinez, L. (2013). Multiresistant Gram-negative bacterial infections: Enterobacteria, Pseudomonas aeruginosa, Acinetobacter baumannii and other non-fermenting Gram-negative bacilli. Enferm. Infecc. Microbiol. Clin. 31, 402-409. doi: 10.1016/j.eimc.2013.03.016

Friese, A., Schulz, J., Laube, H., von Salviati, C., Hartung, J., and Roesler, U. (2013). Faecal occurrence and emissions of livestock-associated methicillinresistant Staphylococcus aureus (laMRSA) and ESbl/AmpC-producing E. coli from animal farms in Germany. Berl. Munch. Tierarztl. Wochenschr. 126, 175-180. doi: 10.2376/0005-9366-126-175

Gilarranz, R., Juan, C., Castillo-Vera, J., Chamizo, F. J., Artiles, F., Alamo, I., et al. (2013). First detection in Europe of the metallo-beta-lactamase IMP-15 in clinical strains of Pseudomonas putida and Pseudomonas aeruginosa. Clin. Microbiol. Infect. 19, E424-E427. doi: 10.1111/1469-0691.12248

Girlich, D., Poirel, L., and Nordmann, P. (2011). Diversity of clavulanic acidinhibited extended-spectrum beta-lactamases in Aeromonas spp. from the Seine River, Paris, France. Antimicrob. Agents Chemother. 55, 1256-1261. doi: 10.1128/AAC.00921-10

\section{SUPPLEMENTARY MATERIAL}

The Supplementary Material for this article can be found online at: http://journal.frontiersin.org/article/10.3389/fmicb. 2016.00586

\section{Supplementary Figure S1 | Figure of the JDS3 River Danube Survey (high} resolution.pdf)

Supplementary Table S1 | Table of all Pseudomonas spp. isolates with resistance pattern. (TZP), Piperacilin/tazobactam; (CAZ), ceftazidime; (FEP), cefepime; (MEM), meropenem; (IPM), imipenem; (AN), amikacin; (GM), gentamicin; (NN), tobramycin; (CIP), ciprofloxacin; (LEV), levofloxacin; and (SXT),

sulfamethoxazole/trimethoprim.

Hess, S., and Gallert, C. (2014). Demonstration of staphylococci with inducible macrolide-lincosamide-streptogramin B (MLSB) resistance in sewage and river water and of the capacity of anhydroerythromycin to induce MLSB. FEMS Microbiol. Ecol. 88, 48-59. doi: 10.1111/1574-6941.12268

Jamal, W., Albert, M. J., and Rotimi, V. O. (2014). Real-time comparative evaluation of bioMerieux VITEK MS versus Bruker Microflex MS, two matrixassisted laser desorption-ionization time-of-flight mass spectrometry systems, for identification of clinically significant bacteria. BMC Microbiol. 14:289-0140289-0. doi: 10.1186/s12866-014-0289-0

JDS 3, I. (2015). Joint Danube Survey 3. A Comprehensive Analysis of Danube Water Quality. Vienna: ICPDR - International Commission for the Protection of the Danube River.

Juan Nicolau, C., and Oliver, A. (2010). Carbapenemases in Pseudomonas spp. Enferm. Infecc. Microbiol. Clin. 28 (Suppl. 1), 19-28. doi: 10.1016/S0213005X(10)70004-5

Kim, S. E., Park, S. H., Park, H. B., Park, K. H., Kim, S. H., Jung, S. I., et al. (2012). Nosocomial Pseudomonas putida Bacteremia: high rates of carbapenem resistance and mortality. Chonnam Med. J. 48, 91-95. doi: 10.4068/cmj.2012.48.2.91

Korzeniewska, E., and Harnisz, M. (2013). Extended-spectrum beta-lactamase (ESBL)-positive Enterobacteriaceae in municipal sewage and their emission to the environment. J. Environ. Manage. 128, 904-911. doi: 10.1016/j.jenvman.2013.06.051

Maravic, A., Skocibusic, M., Cvjetan, S., Samanic, I., Fredotovic, Z., and Puizina, J. (2015). Prevalence and diversity of extended-spectrum-beta-lactamaseproducing Enterobacteriaceae from marine beach waters. Mar. Pollut. Bull. 90, 60-67. doi: 10.1016/j.marpolbul.2014.11.021

Matuschek, E., Brown, D. F., and Kahlmeter, G. (2014). Development of the EUCAST disk diffusion antimicrobial susceptibility testing method and its implementation in routine microbiology laboratories. Clin. Microbiol. Infect. 20, O255-O266. doi: 10.1111/1469-0691.12373

Mazurier, S., Merieau, A., Bergeau, D., Decoin, V., Sperandio, D., Crepin, A., Barbey, C., et al. (2015). Type III secretion system and virulence markers highlight similarities and differences between human- and plant-associated pseudomonads related to Pseudomonas fluorescens and P. putida. Appl. Environ. Microbiol. 81, 2579-2590. doi: 10.1128/aem.04160-14

Mena, K. D., and Gerba, C. P. (2009). Risk assessment of Pseudomonas aeruginosa in water. Rev. Environ. Contam. Toxicol. 201, 71-115. doi: 10.1007/978-1-44190032-6_3

Molina, L., Udaondo, Z., Duque, E., Fernàndez, M., Molina-Santiago, C., Roca, A., Porcel, M., et al. (2014). Antibiotic resistance determinants in a Pseudomonas putida strain isolated from a hospital. PLoS ONE 9:e81604. doi: 10.1371/journal.pone.0081604

Pfeifer, Y., Cullik, A., and Witte, W. (2010). Resistance to cephalosporins and carbapenems in Gram-negative bacterial pathogens. Int. J. Med. Microbiol. 300, 371-379. doi: 10.1016/j.ijmm.2010.04.005

Reinthaler, F. F., Galler, H., Feierl, G., Haas, D., Leitner, E., Mascher, F., et al. (2013). Resistance patterns of Escherichia coli isolated from sewage sludge in comparison with those isolated from human patients in 2000 and 2009. J. Water. Health. 11, 13-20. doi: 10.2166/wh.2012.207

Sengupta, N., Alam, S. I., Kumar, R. B., and Singh, L. (2011). Diversity and antibiotic susceptibility pattern of cultivable anaerobic bacteria from 
soil and sewage samples of India. Infect. Genet. Evol. 11, 64-77. doi: 10.1016/j.meegid.2010.10.009

Suzuki, Y., Kajii, S., Nishiyama, M., and Iguchi, A. (2013). Susceptibility of Pseudomonas aeruginosa isolates collected from river water in Japan to antipseudomonal agents. Sci. Total Environ. 450-451, 148-154. doi: 10.1016/j.scitotenv.2013.02.011

Tacao, M., Correia, A., and Henriques, I. S. (2015). Low prevalence of carbapenemresistant Bacteria in River Water: resistance is mostly related to intrinsic mechanisms. Microb. Drug Resist. 21, 497-506. doi: 10.1089/mdr.2015.0072

Tissera, S., and Lee, S. M. (2013). Isolation of Extended Spectrum beta-lactamase (ESBL) Producing Bacteria from Urban Surface Waters in Malaysia. Malays. J. Med. Sci. 20, 14-22.

Warburton, D. W., Bowen, B., and Konkle, A. (1994). The survival and recovery of Pseudomonas aeruginosa and its effect upon salmonellae in water: methodology to test bottled water in Canada. Can. J. Microbiol. 40, 987-992. doi: $10.1139 / \mathrm{m} 94-158$
Zurfluh, K., Hachler, H., Nuesch-Inderbinen, M., and Stephan, R. (2013). Characteristics of extended-spectrum beta-lactamase- and carbapenemaseproducing Enterobacteriaceae Isolates from rivers and lakes in Switzerland. Appl. Environ. Microbiol. 79, 3021-3026. doi: 10.1128/AEM.00054-13

Conflict of Interest Statement: The authors declare that the research was conducted in the absence of any commercial or financial relationships that could be construed as a potential conflict of interest.

Copyright (C) 2016 Kittinger, Lipp, Baumert, Folli, Koraimann, Toplitsch, Liebmann, Grisold, Farnleitner, Kirschner and Zarfel. This is an open-access article distributed under the terms of the Creative Commons Attribution License (CC BY). The use, distribution or reproduction in other forums is permitted, provided the original author(s) or licensor are credited and that the original publication in this journal is cited, in accordance with accepted academic practice. No use, distribution or reproduction is permitted which does not comply with these terms. 